Projet de note CRAS, calcul scientifique, soumise à O. Pironneau

\title{
Simulations DE L'ÉCOULEMENT TURBUlEnt MARIN AVEC UN MODÈLE DE DÉCONVOLUTION
}

\author{
Anne-Claire Bennis 11, Roger Lewandowski² et Edriss S. Titi 3
}

\section{Résumé}

\begin{abstract}
On introduit une équation de déconvolution qui généralise l'algorithme de Van-Cittert pour des conditions aux limites de type océananique avec vent fixé. On en déduit un modèle de SGE pour lequel on a existence et unicité d'une solution régulière. Nous détaillons un ensemble de simulations numériques qui montrent l'intérêt pratique du modèle.
\end{abstract}

\begin{abstract}
We display a continous equation for the deconvolution process that generalizes the Van Cittert algorithm in the case of oceanic boundary conditions for a given fixed wind. We deduce a LES model for which we have existence and uniqueness of a strong solution. Finally, we display several numerical simulations showing the practical interest of the model.
\end{abstract}

\section{Abridged English version}

A LES model based for the Navier-Stokes equations (NSE), where the convection term is replaced by $\left(H_{N}(\mathbf{u}) \cdot \nabla\right) \mathbf{u}$, was introduced recently in [5]. In this model, $H_{N}(\mathbf{u})$ depends on $\mathbf{u}$ in the following way. For a given $\mathbf{u}$, with $\nabla \cdot \mathbf{u}=0$, we filter $\mathbf{u}$ by solving the Stokes (Helmholtz) like elliptic problem $-\alpha^{2} \Delta \overline{\mathbf{u}}+\overline{\mathbf{u}}+\nabla \pi=\mathbf{u}, \nabla \cdot \overline{\mathbf{u}}=0$ subject to the appropriate boundary conditions, and we then set $A \overline{\mathbf{u}}=\mathbf{u}$. We then follow the Van Cittert's algorithm (see [5]), $\mathbf{u}_{0}=\overline{\mathbf{u}}, \mathbf{u}_{n+1}=\mathbf{u}_{n}+\left\{\overline{\mathbf{u}}-A^{-1} \mathbf{u}_{n}\right\}$ and we set $\mathbf{u}_{N}=H_{N}(\mathbf{u})$ for some $N$. This model makes sense in the case of periodic boundary conditions (see [5]). Nevertheless, we cannot use it in the case of realistic nonhomogeneous boundary conditions, such as those satisfied by oceanic flows with a fixed wind stress at the surface under the rigid lid hypothesis. For this reason a substantial modification is necessary.

Our contribution starts from observing that the Van Cittert algorithm is in fact nothing but a finite difference scheme of a certain evolution equation. Taking $\tau$ as the phony time evolution parameter, this equation for scalar quantities takes the form $-\alpha^{2} \Delta \frac{\partial v}{\partial \tau}+v=u$, $\left.v\right|_{\tau=0}=\bar{u}$. In this work, we make use of this remark to study the case of an oceanic flow satisfying the boundary conditions (2.1), below. Here $\mathbf{x}=(x, y, z) \in \mathbb{R}^{3}, \mathcal{O} \subset \mathbb{R}^{2}$ is a bounded smooth domain, $\Omega=\mathcal{O} \times[-h, 0], \Gamma_{s}=\mathcal{O} \times\{0\}, \Gamma_{l}=\partial \mathcal{O} \times[-h, 0], \Gamma_{b}=\mathcal{O} \times\{-h\}$; $\Gamma=\Gamma_{s} \cup \Gamma_{l} \cup \Gamma_{b}$. Finally $t \in[0, T]$ is the real time parameter. We first introduce the system (2.4)-(2.5). This yields the deconvolution model (2.7). In this model, we do not make the hydrostatic balance assumption in order to capture and model the vertical convection. For the sake of simplicity, we do not consider the Coriolis force, which can be added to (2.7), without causing any difficulties in our analysis. We now give a short description of our results that will be reported in detail in [1]. The space functions are defined in

\footnotetext{
${ }^{1}$ IRMAR, Campus Beaulieu, Université de Rennes I, 35000 RENNES, France, AnneClaire.Bennis@univ-rennes1.fr

${ }^{2}$ IRMAR, Roger.Lewandowski@univ-rennes1.fr, http://perso.univ-rennes1.fr/roger.lewandowski/

${ }^{3}$ Department of Computer Science and Applied Mathematics, Weizmann Institute of Science Rehovot, 76100, ISRAEL, also Department of Mathematics and Department of Mechanical and Aerospace Engineering, University of California, Irvine, CA 92697-3879, USA
} 
(2.2) below. For a given wind stress $\mathbf{V}, \boldsymbol{\psi}=\boldsymbol{\psi}(\mathbf{V})$ is a divergence free vector field which satisfies the boundary conditions (2.1) and is defined by (2.3). Let $\tau>0$ be given, and $\mathbf{u}=\mathbf{u}(t, \mathbf{x})$ with $\nabla \cdot \mathbf{u}=0$. We set $H_{\tau}(\mathbf{u})(t, \mathbf{x})=\mathbf{v}(\tau, t, \mathbf{x})+\boldsymbol{\psi}(\mathbf{V})(\mathbf{x})$ where $\mathbf{v}(\tau, t, \mathbf{x})$ is solution of system (2.4)-(2.5) for a fixed time $t$ (see (2.6) below). Notice that when $\tau=0$, $H_{0}(\mathbf{u})=\overline{\mathbf{u}}$ and therefore our model is precisely the Leray- $\alpha$ model, as considered in [2] and [3]. When $\tau=N$ is an integer, this model remains the same as the one introduced in [5] for the periodic boundary conditions case, and here it is adjusted to satisfy physical boundary conditions. Our main results are

Theorem 0.1 Assume $\mathbf{V} \in H^{3}(\mathcal{O})^{2}$ with a compact support, $\mathbf{u}_{0} \in \mathbf{I H}_{1}$ (see definition (2.2)) and $\mathbf{f} \in C^{0}\left([0, T], \mathbf{H}_{1}\right)$. Let $\tau>0$ be given, Problem (2.7) has a unique strong solution $\left(\mathbf{u}_{\tau}, p_{\tau}\right) \in L^{2}\left([0, T], H^{2}(\Omega)^{3}\right) \times L^{\infty}\left([0, T], H^{1}(\Omega)\right)$ with $\mathbf{u}_{\tau} \in C\left([0, T], L^{2}(\Omega)^{3}\right)$, $\partial_{t} \mathbf{u}_{\tau} \in L^{2}([0, T] \times \Omega)^{3}$.

Theorem 0.2 There exists $\left(\tau_{n}\right)_{n \in N}$, which goes to infinity, when $n$ goes to infinity, and such that the sequence $\left(\mathbf{u}_{\tau_{n}}\right)_{n \in \mathbb{N}}$ converges weakly in $L^{2}\left([0, T], \mathbf{H}_{1}\right)$ and strongly in $L^{2}\left([0, T], \mathbf{I}_{0}\right)$, to a field $\mathbf{u}=\left(\mathbf{u}_{h}, w\right) \in L^{2}\left([0, T], \mathbf{H}_{1}\right) \cap L^{\infty}\left([0, T], L^{2}(\Omega)^{3}\right)$, a Leray-Hopf weak solution to the NSE, with $\partial_{t} \mathbf{u} \in L^{\frac{4}{3}}\left([0, T], \mathbf{I H}_{1}^{\prime}\right)$ and such that for all $\mathbf{v}=\left(\mathbf{v}_{h}, \theta\right) \in$ $L^{4}\left([0, T], \mathbf{I H}_{1}\right)$ (2.8) is satisfied. Moreover, $\mathbf{u}(t, \cdot)$ converges weakly to $\mathbf{u}_{0}$ in $L^{2}(\Omega)^{3}$ as $t$ goes to 0 . Moreover, $\mathbf{u}$ satisfies the energy inequality.

Finally, we display numerical tests by using FreeFem++ 4 in the 2D case. Our code is semi-implicit and makes use of a method of the caracteristics adjusted to the deconvolution. Two cases have been considered. For each case, the wind stress is equal to $\mathbf{V}(x)=$ $a(x) \sin (\pi x), \mathbf{x}=(x, y), \mathbf{x}_{h}=x \in \Gamma_{s} \approx[0,1]$, where $a(x)$ has a compact support and it localizes the stress. In the first case, $\Omega$ is a square, $\Delta t=0.2 \mathrm{~s}, \alpha=0.1 \mathrm{~m}, T=90 \mathrm{~s}$. The DNS exhibits a good behavior. We compare the cases where the order of deconvolution $\tau$ takes for values $\tau=0$ (Leray- $\alpha$ model), $\tau=5$ and $\tau=20, \Delta \tau=1$. Vertical profils are displayed in Figure 3.1. They confirm the convergence Theorem and the validity of the code. The second case is the one where $\Omega$ has a topography (see Figure 3.2 ),$\Delta t=0.02 \mathrm{~s}$, $\alpha=0.1 \mathrm{~m}, T=10 \mathrm{~s}, \tau=5, \Delta \tau=1$. Poiseuille Flows have been imposed on the lateral boundaries to enhance turbulence and the DNS converges very slowly. Figure 3.3 displays one profil and underlines the improvement of the computation using the model compared with the Leray- $\alpha$ model. Therefore, our model appears to produce good results and hence could be suggested as a good alternative for simulations in real situations.

\section{Introduction}

On a introduit dans [5] un modèle de SGE basé sur les équations de Navier-Stokes, dans lequel le terme de convection vaut $\left(H_{N}(\mathbf{u}) \cdot \nabla\right) \mathbf{u}, H_{N}(\mathbf{u})$ étant déduit de la vitesse $\mathbf{u}$ (avec $\nabla \cdot \mathbf{u}=0$ ) en partant de la filtration $-\alpha^{2} \Delta \overline{\mathbf{u}}+\overline{\mathbf{u}}+\nabla \pi=\mathbf{u}, \nabla \cdot \overline{\mathbf{u}}=0$. On pose $A \overline{\mathbf{u}}=\mathbf{u}$, que l'on déconvole à l'aide de l'algorithme de Van Cittert, $\mathbf{u}_{0}=\overline{\mathbf{u}}, \mathbf{u}_{n+1}=$ $\mathbf{u}_{n}+\left\{\overline{\mathbf{u}}-A^{-1} \mathbf{u}_{n}\right\}, \mathbf{u}_{N}=H_{N}(\mathbf{u})$. Ceci a un sens dans le cas de conditions aux limites périodiques et permet d'approcher les équations de Navier-Stokes par des problèmes bien posés avec un bon contrôle de l'erreur quand les lois de Kolmogorov sont satisfaites.

Il semble difficile d'appliquer cette procédure telle quelle dans le cas de conditions aux limites réalistes, comme par exemple pour l'Océan avec une tension du vent en surface fixée, sous l'hypothèse du toît rigide. La remarque principale dans ce travail est que l'algorithme de Van Cittert peut être vu comme l'équation aux différences finies d'une

équation d'évolution qui s'écrit pour des scalaires sous la forme $-\alpha^{2} \Delta \frac{\partial v}{\partial \tau}+v=u,\left.u\right|_{\tau=0}=$ 
$\bar{u}$, où $\tau$ est le paramètre de déconvolution. Nous exploitons cette remarque de manière rigoureuse pour les conditions aux limites (2.1) satisfaites par la vitesse de l'écoulement marin, en introduisant le système (2.4)-(2.5) . Cela nous permet d'introduire le modèle de SGE (2.7). Dans ce modèle, nous ne faisons pas l'hypothèse hydrostatique pour mieux décrire les effets convectifs et capter la couche de mélange. Pour simplifier la présentation, nous n'avons pas pris en compte la force de Coriolis, qui peut être rajoutée dans (2.7) sans changer la nature de notre analyse.

Nous montrons que ce modèle conduit à un problème bien posé et nous avons un résultat de convergence vers les équations de Navier-Stokes quand le paramètre de déconvolution tends vers l'infini. Dans une série de tests numériques, nous validons le modèle dans le cas 2D en comparant les résultats obtenus avec ceux d'une DNS convergente. Nous produisons des calculs numériques dans un cas avec topographie, cas pour lequel la DNS a un mauvais comportement. Le modèle donne des bons résultats numériques pour $\tau=5$ qui affinent ceux donnés par les Leray- $\alpha$ modèles habituels adaptés à nos conditions aux limites avec notre méthode, puisqu'ils correspondent au cas $\tau=0$ (voir les modèles d'origine dans [2], [3]). Cette note annonce des résultats développés dans l'article en cours de finalisation [1].

\section{Resultats théoriques}

\subsection{Géométrie et espaces fonctionnels}

On note $\mathbf{x}=(x, y, z)$ un point de $\mathbb{R}^{3}, \mathcal{O} \subset \mathbb{R}^{2}$ un domaine borné régulier de $\mathbb{R}^{2}, \Omega=$ $\mathcal{O} \times[-h, 0]$, où $h>0$. Soient $\Gamma_{s}$ la surface, $\Gamma_{s}=\mathcal{O} \times\{0\}$, et $\Gamma_{l}$ et $\Gamma_{b}$ les frontières latérales et le fond, $\Gamma_{l}=\partial \mathcal{O} \times[-h, 0], \quad \Gamma_{b}=\mathcal{O} \times\{-h\}$. On note enfin $\Gamma=\Gamma_{s} \cup \Gamma_{l} \cup \Gamma_{b}$.

Soit $\mathbf{u}=\left(\mathbf{u}_{h}, w\right)$ la vitesse de l'eau de mer, $\mathbf{u}_{h}=(u, v)$ sa partie horizontale. Les conditions aux limites classiques sont (sous l'hypothèse du toît rigide)

$$
\left.w\right|_{\Gamma}=0,\left.\quad \mathbf{u}_{h}\right|_{\Gamma_{l} \cup \Gamma_{b}}=0,\left.\quad \nabla \mathbf{u}_{h} \cdot \mathbf{n}\right|_{\Gamma_{s}}=\mathbf{V},
$$

où $\mathbf{n}$ est la normale sortante, $\mathbf{V}=\mathbf{V}(x, y)$ est le vent (ici fixé) défini sur $\mathcal{O}$. On identifie $\mathcal{O}$ et $\Gamma_{s}$. Les espaces fonctionnels sont les suivants :

$$
\begin{aligned}
& \mathbf{I H}_{0}=\left\{\mathbf{u}=\left(\mathbf{u}_{h}, w\right) \in L^{2}(\Omega)^{3} ;\left.\mathbf{u} \cdot \mathbf{n}\right|_{\Gamma}=0, \nabla \cdot \mathbf{u}=0\right\} \\
& \mathbf{I H}_{1}=\left\{\mathbf{u}=\left(\mathbf{u}_{h}, w\right) \in H^{1}(\Omega)^{3},\left.w\right|_{\Gamma}=0,\left.\mathbf{u}_{h}\right|_{\Gamma_{l} \cup \Gamma_{b}}=\mathbf{0}, \nabla \cdot \mathbf{u}=0\right\}
\end{aligned}
$$

Nous montrons dans [1] l'existence d'une base spéciale adaptée, résumé dans ce qui suit.

Lemme 2.1 Il existe une suite de réels strictement positifs $\left(\lambda_{n}\right)_{n \in \mathbb{N}}$ des suites $\left(\mathbf{e}_{n}\right)_{n \in \mathbb{N}}$, $\left(\pi_{n}\right)_{n \in \mathbb{N}}$ où $\mathbf{e}_{n} \in \mathbf{I H}_{1} \cap H^{2}(\Omega)^{3}, \pi_{n} \in H^{1}(\Omega)$ pour chaque $n$ et tels que $-\Delta \mathbf{e}_{n}+\nabla \pi_{n}=\lambda_{n} \mathbf{e}_{n}$.

La démonstration utilise un argument de symétrie pour se ramener un problème de Stokes sur un cylindre avec des conditions aux limites de Dirichlet homogènes. Un résultat analogue avec des équations primitives a déjà été prouvé dans [7] avec la même technique. Nous montrons dans [1] que l'injection $\mathbf{I H}_{1} \subset \mathbf{I H}_{0}$ est dense. Nous terminons cette section par le relèvement de la condition aux limites. On suppose que $\mathbf{V}=\mathbf{V}(x, y)$ est à support compact dans $\Gamma_{s}$. Soit $\boldsymbol{\psi}=\boldsymbol{\psi}(x, y, z)$ le champ de vecteurs défini dans $\Omega$ par

$$
\boldsymbol{\psi}(x, y, z)=\left(\begin{array}{l}
\psi_{h} \\
\psi_{v}
\end{array}\right)=\left(\begin{array}{l}
\rho(z) \mathbf{V}(x, y) \\
-\kappa(z)\left(\nabla_{h} \cdot \mathbf{V}\right)(x, y)
\end{array}\right)=\boldsymbol{\psi}(\mathbf{V})
$$

où $\kappa(z)=\int_{-h}^{z} \rho\left(z^{\prime}\right) d z^{\prime}$ et $\rho(z)=\frac{3}{4 h} z^{2}+z+\frac{h}{4}$. Il est facile de vérifier que $\boldsymbol{\psi}=\boldsymbol{\psi}(\mathbf{V})$ est à divergence nulle dans $\Omega$ et satisfait les conditions aux limites (2.1). 


\subsection{La Filtration}

On suppose que $\mathbf{V} \in H^{3}(\mathcal{O})^{2}$ et est à support compact, $\alpha>0$ est fixé, $\mathbf{u} \in \mathbf{I H}_{1}$. Soit

$$
\left\{\begin{array}{l}
-\alpha^{2} \Delta \hat{\overline{\mathbf{u}}}+\hat{\overline{\mathbf{u}}}+\nabla r=\mathbf{u}-\boldsymbol{\psi}(\mathbf{V}), \quad \nabla \cdot \hat{\overline{\mathbf{u}}}=0 \\
\left.\hat{\bar{w}}\right|_{\Gamma}=0,\left.\quad \hat{\mathbf{u}}_{h}\right|_{\Gamma_{l} \cup \Gamma_{b}}=0,\left.\quad \nabla \hat{\mathbf{u}}_{h} \cdot \mathbf{n}\right|_{\Gamma_{s}}=\mathbf{0}
\end{array}\right.
$$

En raisonnant par symétrisation on montre dans [1] que (2.4) admet une unique solution $(\hat{\overline{\mathbf{u}}}, r)$ avec $\hat{\overline{\mathbf{u}}} \in H^{3-\varepsilon}(\Omega)^{3}$ pour tout $\varepsilon>0$. Soit $\overline{\mathbf{u}}$ défini par $\overline{\mathbf{u}}=\hat{\overline{\mathbf{u}}}+\boldsymbol{\psi}(\mathbf{V})$. On note que $\overline{\mathbf{u}}$ a une trace sur $\Gamma_{s}$ ainsi que $\nabla \overline{\mathbf{u}}$ et donc $\overline{\mathbf{u}}$ satisfait (2.1). Soit $\mathbf{u}=\mathbf{u}(t, \mathbf{x})$ à divergence nulle. L'équation de déconvolution est, où $\tau$ est le paramètre de déconvolution et $\mathbf{v}=\mathbf{v}(\tau, t, \mathbf{x})=\left(\mathbf{v}_{h}, \theta\right), t \in[0, T]$ est fixé,

$$
\left\{\begin{array}{l}
-\alpha^{2} \Delta \frac{\partial \mathbf{v}}{\partial \tau}+\mathbf{v}+\nabla \pi=\mathbf{u}-\boldsymbol{\psi}(\mathbf{V})=\hat{\mathbf{u}}, \quad \nabla \cdot \mathbf{v}=0 \\
\left.\mathbf{v}_{h}\right|_{\Gamma_{l} \cup \Gamma_{b}}=\mathbf{0},\left.\quad \nabla \mathbf{v}_{h} \cdot \mathbf{n}\right|_{\Gamma_{s}}=\mathbf{0},\left.\quad \theta\right|_{\partial \Omega}=0, \quad \mathbf{v}(0, \mathbf{x})=\overline{\mathbf{u}}+\boldsymbol{\psi}(\mathbf{V})=\hat{\overline{\mathbf{u}}}
\end{array}\right.
$$

Soit $\tau>0, H_{\tau}(\mathbf{u})(t, \mathbf{x})=\mathbf{v}(\tau, t, \mathbf{x})+\boldsymbol{\psi}(\mathbf{V})(\mathbf{x})$. Avec la base du Lemme 2.1, on écrit pour $\mathbf{u} \in L^{2}\left([0, T], \mathbf{H}_{1}\right) \cap L^{\infty}\left([0, T], \mathbf{H}_{0}\right), \mathbf{u}=\sum_{k=1}^{\infty} u_{k}(t) \mathbf{e}_{k}$ et $\boldsymbol{\psi}(\mathbf{V})=\sum_{k=1}^{\infty} \psi_{k} \mathbf{e}_{k}$. On a

$$
H_{\tau}(\mathbf{u})(t, \mathbf{x})=\mathbf{u}(t, \mathbf{x})+\sum_{k=1}^{\infty}\left(\bar{u}_{k}(t)-u_{k}(t)\right) \exp \left(-\frac{\tau}{\alpha^{2} \lambda_{k}}\right) \mathbf{e}_{k}(\mathbf{x}), \bar{u}_{k}(t)=\frac{u_{k}(t)+\alpha^{2} \psi_{k}}{1+\alpha^{2} \lambda_{k}}
$$

\subsection{Le modèle de déconvolution}

Le modèle de SGE que nous considérons est le suivant, avec $\mathbf{u}$ la vitesse et $p$ la pression :

$$
\left\{\begin{array}{l}
\partial_{t} \mathbf{u}+\left(H_{\tau}(\mathbf{u}) \cdot \nabla\right) \mathbf{u}-\nu \triangle \mathbf{u}+\nabla p=\mathbf{f}, \quad \nabla \cdot \mathbf{u}=0, \\
\left.w\right|_{\Gamma}=0,\left.\quad \mathbf{u}_{h}\right|_{\Gamma_{l} \cup \Gamma_{b}}=0,\left.\quad \nabla \mathbf{u}_{h} \cdot \mathbf{n}\right|_{\Gamma_{s}}=\mathbf{V}, \quad \mathbf{u}_{t=0}=\mathbf{u}_{0},
\end{array}\right.
$$

Notons que quand $\tau=0$, il s'agit du Leray- $\alpha$ modèle ([2]). Nous prouvons dans [1] :

Théorème 2.1 Soit $\mathbf{V} \in H^{3}(\mathcal{O})^{2}$ à support compact, $\mathbf{u}_{0} \in \mathbf{I H}_{1}$ et $\mathbf{f} \in C^{0}\left([0, T], \mathbf{H}_{1}\right)$. Pour $\tau>0$ fixé, le système (2.7) admet une unique solution $\left(\mathbf{u}_{\tau}, p_{\tau}\right) \in L^{2}\left([0, T], H^{2}(\Omega)^{3}\right)$ $\times L^{\infty}\left([0, T], H^{1}(\Omega)\right)$ avec $\mathbf{u}_{\tau}=\mathbf{u}_{\tau}(t, \mathbf{x}) \in C\left([0, T], L^{2}(\Omega)^{3}\right), \partial_{t} \mathbf{u}_{\tau} \in L^{2}([0, T] \times \Omega)^{3}$.

Théorème 2.2 Il existe une suite $\left(\tau_{n}\right)_{n \in N}$ qui tend vers l'infini quand $n$ tend vers l'infini, telle que $\left(\mathbf{u}_{\tau_{n}}\right)_{n \in \mathbb{N}}$ converge faiblement dans $L^{2}\left([0, T], \mathbf{H}_{1}\right)$, fortement dans $L^{2}\left([0, T], \mathbf{I}_{0}\right)$, vers un champ $\mathbf{u}=\left(\mathbf{u}_{h}, w\right) \in L^{2}\left([0, T], \mathbf{I H}_{1}\right) \cap L^{\infty}\left([0, T], L^{2}(\Omega)^{3}\right)$ avec $\partial_{t} \mathbf{u} \in L^{\frac{4}{3}}\left([0, T], \mathbf{H}_{1}^{\prime}\right)$ et tel que pour chaque $\mathbf{v}=\left(\mathbf{v}_{h}, \theta\right) \in L^{4}\left([0, T], \mathbf{H}_{1}\right)$ on a

$$
\left\langle\partial_{t} \mathbf{u}, \mathbf{v}>-\int_{0}^{T} \int_{\Omega} \mathbf{u} \otimes \mathbf{u}: \nabla \mathbf{v}+\nu \int_{0}^{T} \int_{\Omega} \nabla \mathbf{u}: \nabla \mathbf{v}-\int_{0}^{T} \int_{\Gamma_{s}} \mathbf{V} \cdot \mathbf{v}_{h}=\int_{0}^{T} \int_{\Omega} \mathbf{f} \cdot \mathbf{v} .\right.
$$

De plus, $\mathbf{u}(t, \cdot)$ tend faiblement vers $\mathbf{u}_{0}$ dans $L^{2}(\Omega)^{3}$ quand $t$ tend vers 0 , et vérifie l'inégalité d'énergie.

\section{Résultats numériques}

On résoud (2.7) par la méthode des éléments finis en utilisant FreeFem ++ [4 dans le cas 2D, pour $\tau$ fixé. On détermine la pression et la vitesse par un schéma semi-implicite initialisé avec des solutions d'un problème de Stokes. On a écrit un algorithme de résolution itératif qui adapte la méthode des caractéristiques au cas du transport déconvolé. On arrête la simulation lorsque les algorithmes ont convergé. On présente un premier cas servant à valider notre modèle de déconvolution et un deuxième cas qui montre son intérêt pratique. 


\subsection{Turbulence induite par le vent de surface}

On considère $\Omega=[0,1] \times[-0.5,0]$, triangulé avec $h_{\max }=0.014 \mathrm{~m}$ et $h_{\min }=0.006 \mathrm{~m}$. On choisit $\mathbf{V}(x)=a(x) \sin (\pi x)$ où $a(x)$ est une fonction de localisation à support compact. On compare les résultats des simulations DNS et LES pour des paramètres de déconvoution successivement égaux à $\tau=0, \tau=5$ ou $\tau=20$ grâce à des profils verticaux ; ici $\Delta \tau=1$, $\alpha=0.1 \mathrm{~m}, \Delta t=0.2 \mathrm{~s}$ et on a fait 450 itérations en temps, ce qui nous donne le résultat pour $T=90 \mathrm{~s}$. On trouve une erreur $L_{2}$ moyenne de $0.5 \%$ entre les résultats DNS et les résultats du modèle de déconvolution pour $\tau=20$ et de $1 \%$ pour $\tau=5$, ce qui est satisfaisant et ce qui valide notre modélisation. De plus, on a divisé l'erreur $L_{2}$ moyenne d'un facteur 4 entre les résultats du Leray- $\alpha$ modèle $(\tau=0)$ et ceux du modèle de déconvolution pour $\tau=20$, ce qui montre l'amélioration apportée.
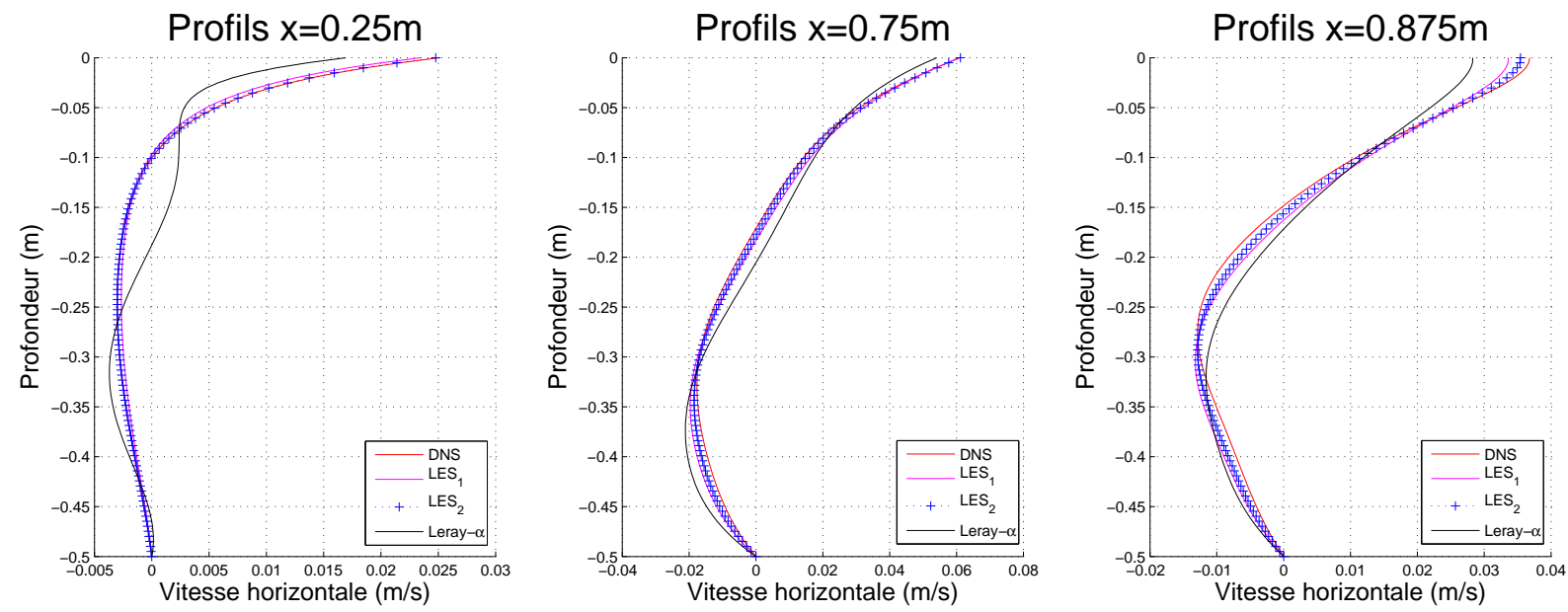

Figure 3.1: Profils verticaux de la vitesse horizontale pour la DNS (DNS), pour le modèle de déconvolution avec $\tau=5$ (LES-1) et avec $\tau=20$ (LES-2) et pour le modèle Leray- $\alpha$ (Leray- $\alpha$ )

\subsection{Cas avec bathymétrie}

On considère le domaine $\Omega$ décrit ci-dessous avec une bosse, triangulé avec $h_{\max }=0.010 \mathrm{~m}$ et $h_{\min }=0.003 \mathrm{~m}$.
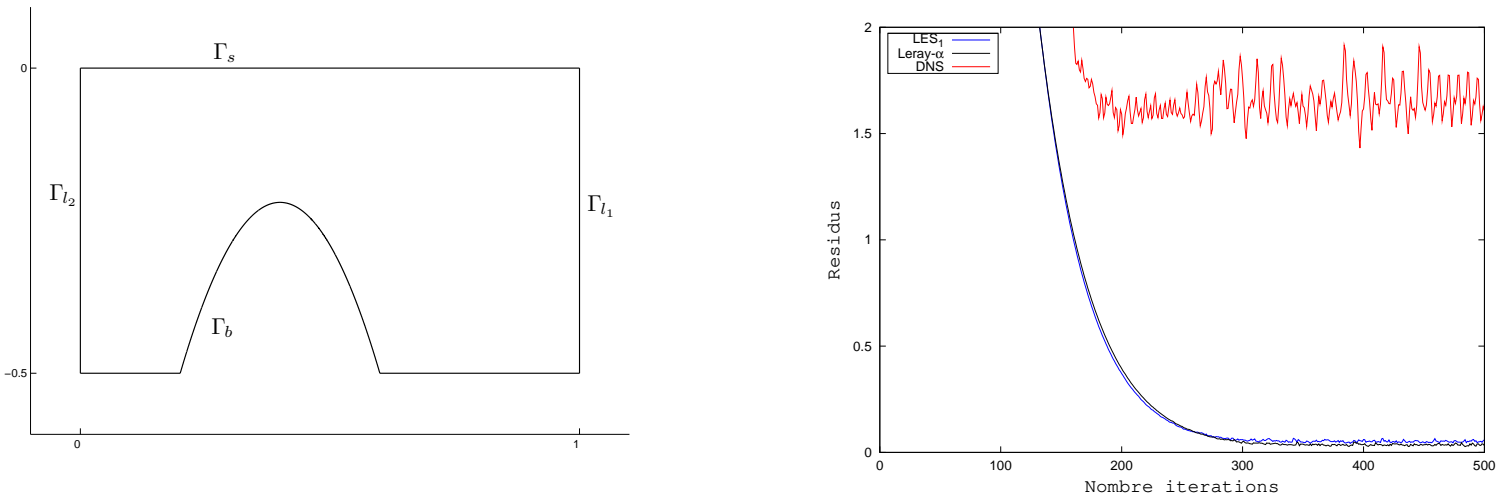

Figure 3.2: Géométrie du domaine à gauche et Résidus des équations de Navier-Stokes à droite (DNS en rouge, modèle de déconvolution en bleu et modèle Leray- $\alpha$ en noir) 
On prend le même vent à la surface que précédemment et on impose un flot de poiseuille sur les bords d'entrée et de sortie $\Gamma_{l_{1}}$ et $\Gamma_{l_{2}}$ afin d'augmenter le niveau de turbulence. On conserve les paramètres $\alpha=0.1 \mathrm{~m}, \Delta t=0.02 \mathrm{~s}, \tau=5$ et on fait 500 itérations en temps, soit $T=10 \mathrm{~s}$. On observe que la DNS converge difficilement avec des résidus élevés, ce qui n'est pas le cas des modèles LES (voir Figure 3.2).

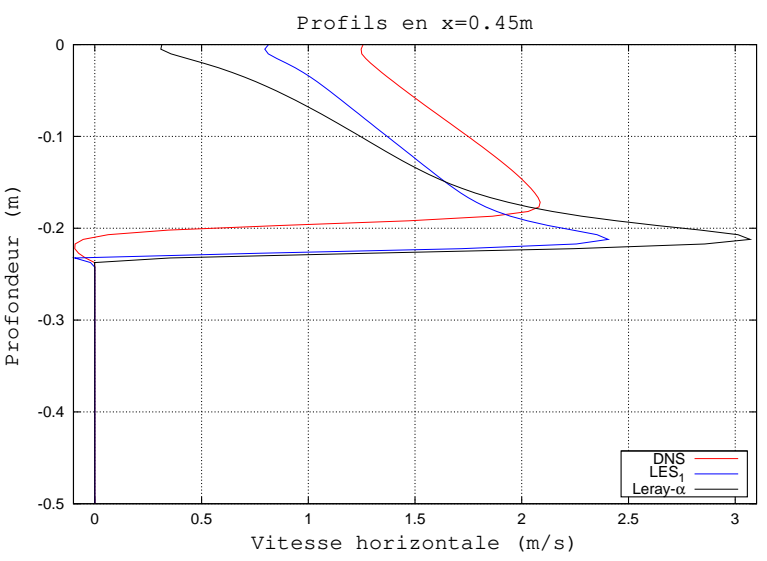

Figure 3.3: Profil vertical de la vitesse horizontale. Modèle de déconvolution en bleu (LES-1), modèle Leray- $\alpha$ en noir (Leray- $\alpha$ ) et DNS en rouge
On observe que notre modèle améliore la solution donnée par le modèle Leray$\alpha$, ce qui est en accord avec les conclusions précédentes. Ce profil montre qu'il faut augmenter le nombre de déconvolution qui est égal à 5 comme préconisé par Adams et al [6]. Il est aussi nécessaire d'abaisser la valeur du paramètre de filtration $\alpha$, afin d'avoir un temps de calcul raisonnable par un abaissement du taux de déconvolution. Ce cas montre que notre modèle peut corriger les effets de la filtration et montre aussi que le calage des paramètres de déconvolution et de filtration n'est pas le même selon les cas étudiés.

Aknowledgement. The work of Roger Lewandowski is partially supported by the ANR project 08FA300-01. The work of Edriss S. Titi is partially supported by NSF grant No DMS-0708832 and the ISF Grant No 120/6.

\section{References}

[1] A.-C. Bennis, R. Lewandowski, And E. S. Titi, A generalized Leray-deconvolution model of turbulence, In Preparation, (2008).

[2] A. Cheskidov, D. D. Holm, E. Olson, And E. S. Titi, On a Leray- $\alpha$ model of turbulence, Royal Society London, Proceedings, Series A, Mathematical, Physical and Engineering Sciences, 461 (2005), pp. 629-649.

[3] B. J. Geurts And D. D. Holm, Regularization modeling for large eddy simulation, Physics of fluids, 15 (2003), pp. L13-L16.

[4] F. Hecht, O. Pironneau, A. L. Hyaric, and K. Ohtsua, Freefem ++ manual v2.21, (2006).

[5] W. Layton and R. Lewandowski, A high accuracy Leray-deconvolution model of turbulence and its limiting behavior, Analysis and Applications, 6 (2008), pp. 1-27.

[6] S. Stolz, N. A. Adams, And L. Kleiser, An approximate deconvolution model for large-eddy simulation with application to incompressible wall-bounded flows, Physics of fluids, 13 (2001), pp. 997-1015.

[7] M. Ziane, Regularity results for a Stokes type system, Applicable Aanalysis, 58 (1995), pp. 263-293. 


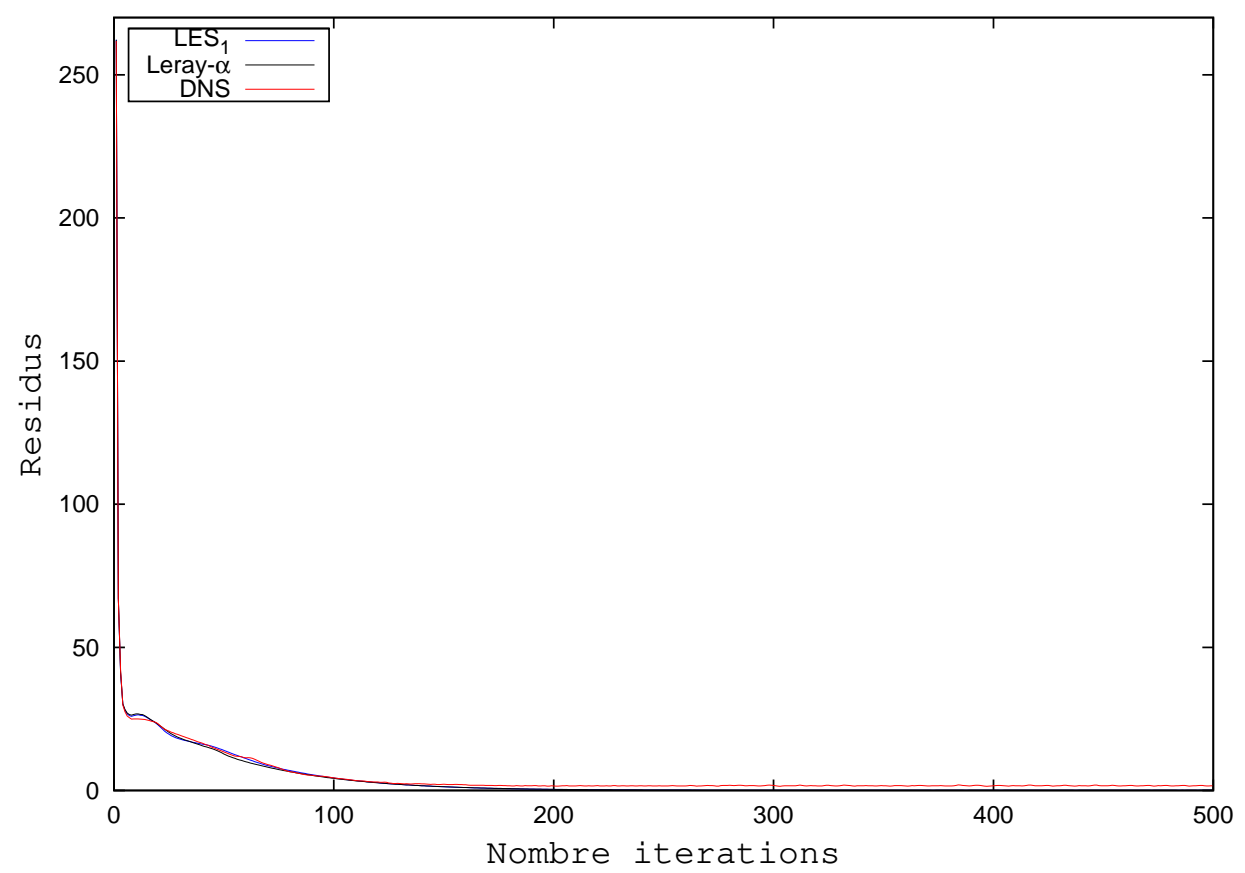

